\title{
Spherical shock waves in general relativity
}

\author{
Y. Nutku \\ Department of Mathematics, Bilkent University, 06533 Bilkent, Ankara, Turkey
}

(Received 21 May 1990)

\begin{abstract}
We present the metric appropriate to a spherical shock wave in the framework of general relativity. This is a Petrov type- $N$ vacuum solution of the Einstein field equations where the metric is continuous across the shock and the Riemann tensor suffers a step-function discontinuity. Spherical gravitational waves are described by type- $N$ Robinson-Trautman metrics. However, for shock waves the RobinsonTrautman solutions are unacceptable because the metric becomes discontinuous in the RobinsonTrautman coordinate system. Other coordinate systems that have so far been introduced for describing Robinson-Trautman solutions also suffer from the same defect. We shall present the $C^{0}$-form of the metric appropriate to spherical shock waves using Penrose's approach of identification with warp. Further extensions of Penrose's method yield accelerating, as well as coupled electromagnetic-gravitational shock-wave solutions.
\end{abstract}

\section{INTRODUCTION}

The characteristic property of gravitational shock waves is a step-function discontinuity in the curvature of type- $N$ vacuum solutions of the Einstein field equations [1]. Since the abstract metric tensor is continuous, the principal problem in dealing with gravitational shock waves lies in the construction of a coordinate system whereby the continuity of the metric across the shock is manifest. For plane waves this problem of finding the $C^{0}$-form of the metric was solved two decades ago. It proved to be a milestone in the solution of further problems of physical interest such as colliding impulsive gravitational waves $[2,3]$ and various combinations of impulsive and shock waves $[4,5]$ with plane wave fronts. The lack of progress in problems involving spherical waves can be traced back to the unavailability of the $C^{0}$-form of the metric. We shall now present the continuous form of the metric appropriate to a spherical shock wave, cf. Eqs. (8), (9), and (10) below.

Exact solutions of the Einstein field equations describing spherical gravitational waves were first obtained by Robinson and Trautman [6,7]. Newman and Unti [8] have given an interpretation of these solutions as the gravitational radiation field of an accelerating particle. Penrose [1] has remarked that the Robinson-Trautman form of the metric is inadequate for describing spherical impulsive, or shock waves. Type- $N$ Robinson-Trautman solutions contain an arbitrary function $f$ of the retarded time, cf. Eq. (24) below, which can be chosen to fit the profile of the wave. The limiting form of this function will be a distribution, a Dirac $\delta$ function for impulsive waves, or a Heaviside step function for shock waves. In either case the Robinson-Trautman form of the metric is undefined, as a glance at Eqs. (24) shows that at best the metric will be $C^{-1}$. An alternative expression for the Robinson-Trautman solutions is the Foster-Newman [9] form, which is also discontinuous in the case of shock waves and therefore unacceptable as a metric.
Penrose $[1,10]$ has developed a "scissors and paste" approach for handling distribution-valued metrics, which will be referred to as identification with warp. Most recently this method has been used to construct the $C^{0}$ form of the metric appropriate to impulsive spherical waves [11]. The resulting solution has been interpreted as the gravitational radiation field of a snapping cosmic string $[12,13]$. Type- $N$ Robinson-Trautman solutions have an interesting singularity structure owing to the fact that in general relativity spherical wave fronts must necessarily be incomplete because monopole radiation is forbidden by the principle of equivalence. The wire singularities of the Robinson-Trautman solutions fit very naturally into the snapping string interpretation. The quantum effects in the background of this solution were studied by Hortaçsu [14]. The metric of a snapping cosmic string which corresponds to the particular case of an exponential warp function for the impulsive spherical wave solutions was discussed by Gleiser and Pullin [15].

We shall now show that an application of Penrose's approach yields the $C^{0}$-metric for a spherical shock wave. We shall also present new exact solutions describing accelerating, as well as coupled gravitational and electromagnetic spherical shock waves which are obtained by direct extensions of Penrose's method of identification with warp. All our considerations will be local.

\section{IDENTIFICATION WITH WARP}

Penrose's construction of a spherical gravitational wave starts with the removal of a null cone from Minkowski space (cf. Fig. 3 in Ref. [1]). The coordinates suitable for this purpose are derived from the null system with the metric

$$
d s^{2}=2 d u^{\prime} d v^{\prime}-2 d \zeta^{\prime} d \bar{\zeta}^{\prime}
$$

according to the transformation 


$$
\begin{aligned}
& v^{\prime}=v+\frac{u}{P}|\zeta|^{2}, \\
& u^{\prime}=\frac{k}{2} v+\frac{u}{P}, \\
& \zeta^{\prime}=\frac{u}{P} \zeta
\end{aligned}
$$

where

$$
\begin{aligned}
& k= \pm 1,0, \\
& P=1+\frac{k}{2}|\xi|^{2} .
\end{aligned}
$$

As a result of this transformation the Minkowski metric becomes

$$
d s^{2}=2 d u d v+k d v^{2}-2 \frac{u^{2}}{P^{2}} d \xi d \bar{\zeta},
$$

where $v$ is a null coordinate which can be regarded as retarded time, $u$ is a Bondi-type luminosity distance, and $\zeta$ is the stereographic coordinate on the sphere.

Now the hypersurface $N$ defined by $v=0$ is a null cone and its removal leaves two disjoint pieces $M^{-}, M^{+}$which are given by $v<0, v>0$ respectively. If we reattach $M^{-}$ and $\mathrm{M}^{+}$along $N$, identifying in warped fashion,

$$
\{u, 0, \zeta, \bar{\zeta}\}_{-}=\left\{\frac{u P[h(\zeta)]}{\left|h_{\zeta}\right|}, 0, h(\zeta), \bar{h}(\bar{\zeta})\right\}_{+}
$$

we find that

$$
h_{\bar{\xi}}=0
$$

is sufficient to guarantee the satisfaction of the Einstein field equations. For $k=0$ Eqs. (6) reduce to Penrose's identification [1].

We shall first present the continuous form of the metric for a spherical shock wave that is obtained from the above process of identification with warp and then comment on its derivation. The explicit form of the metric which results from the identification of $M^{-}$and $M^{+}$according to the warp given by Eqs. (6) is

$$
\begin{aligned}
d s^{2}= & 2 \mathcal{P} d u d v+2 u \mathcal{P}_{\zeta} d \xi d v+2 u \mathcal{P}_{\bar{\zeta}} d \bar{\zeta} d v \\
& +k d v^{2}-2 u^{2} d \zeta d \bar{\zeta}
\end{aligned}
$$

where

$$
\mathscr{P}=\frac{1+(k / 2)|h|^{2}}{\left|h_{\zeta}\right|}
$$

and the warp function

$$
h=h(\zeta+v \theta(v))
$$

is an arbitrary holomorphic function of its argument with $\theta$ standing for the Heaviside unit step function. The continuity of the metric (8) follows from the continuity of $h$ across $v=0$. The calculation of the curvature of this metric in the next section shows that the Riemann tensor suffers the $\theta$ function discontinuity that is expected of a pure shock wave.

To check the derivation of Eq. (8) we shall first restrict our attention to the flat manifold $M^{-}$where $v<0$. Then Eqs. (6) can be regarded as a coordinate transformation on flat space that takes the Minkowski metric in the form of Eq. (5) into the form of Eq. (8). This calculation, which is readily verified, establishes the general form of the metric in Eqs. (8) and (9). We had seen that condition (7) requiring $h$ to be a holomorphic function is sufficient to guarantee the satisfaction of the Einstein field equations. In the process of identification with warp we must retain this condition while allowing for the warp function $h$ to assume a different dependence on $v$ in $M^{-}$and $M^{+}$. Since $M^{-}$is to remain flat we are led to

$$
h=h(\zeta+F(v)),
$$

where apart from the requirement

$$
\left.F(v)\right|_{v<\text { const }}=0
$$

$F$ can be an arbitrary function of $v$. If we were to take this form of the metric as an ansatz, then $F$ could generally be written as

$$
F(v)=\sum_{n=1}^{\infty} c_{n}\left(v-v_{0_{n}}\right)^{n} \theta\left(v-v_{0_{n}}\right)
$$

where $c_{n}$ and $v_{0_{n}}$ are arbitrary constants. Waves traveling in the same direction can be superposed, as evidenced by the existence of the constants $v_{0}$ above. The polarization degree of freedom of the gravitational wave finds its expression in the existence of infinitely many arbitrary coefficients $c_{n}$ which can be chosen to fit the profile of the wave. However, the dependence of $h$ on $v$ is such that the Riemann tensor [cf. Eq. (15)] will suffer a step discontinuity only for the case $n=1$. Since the Riemann tensor is going to be continuous for $n>1$, we shall henceforth confine our attention solely to the case $n=1$, i.e., Eq. (7), which yields the shock-wave solution.

Finally, we note that in the above expression for $F$ the sum over $n$ starts with one. The case $n=0$ would have corresponded to an impulsive wave, but this is unacceptable because the metric would then have been discontinuous. The precise nature of the solution which results from an identification with warp depends, of course, on the identification itself. The choice in Eqs. (6) is the one appropriate to shock waves and results in a curved $M^{+}$. The warped identification that results in an impulsive spherical wave, where both $M^{-}$and $M^{+}$are flat even after the identification, requires a modification of Eqs. (6) as in Ref. [11].

\section{CURVATURE}

In order to elucidate the properties of the metric (8) and show that it describes a spherical gravitational shock wave, we need to calculate its curvature. For this purpose we shall use the Newman-Penrose [16] (NP) formalism. The metric (8) can be written in the form

$$
d s^{2}=l \otimes n+n \otimes l-m \otimes \bar{m}-\bar{m} \otimes m
$$

with the null coframe 


$$
\begin{aligned}
& l=d v, \\
& n=\frac{k}{2} d v+\mathcal{P} d u+u \mathcal{P}_{\zeta} d \zeta+u \mathcal{P}_{\bar{\zeta}} d \bar{\zeta}, \\
& m=u d \xi .
\end{aligned}
$$

For this null tetrad the NP spin coefficients are given by

$$
\begin{aligned}
& \sigma=\kappa=\lambda=\epsilon=\pi=\tau=0, \\
& \rho=-\frac{1}{u \mathscr{P}}, \\
& \mu=-\frac{k}{2 u \mathcal{P}}, \\
& \gamma=-\frac{1}{2 \mathcal{P}} \mathcal{P}_{v}, \\
& \alpha=-\frac{1}{2 u \mathscr{P}} \mathscr{P}_{\zeta}, \\
& \beta=\frac{1}{2 u \mathcal{P}} \mathcal{P}_{\bar{\zeta}}, \\
& \nu=\frac{1}{\mathcal{P}^{3}}\left(\mathcal{P P}_{\xi v}-\mathcal{P}_{\xi} \mathcal{P}_{v}\right)
\end{aligned}
$$

and from the vanishing of $\sigma$ we see that the hypersurfaces $v=$ const are shear free. The Einstein field equations reduce to Liouville's equation

$$
\mathcal{P P}_{\zeta \bar{\xi}}-\mathcal{P}_{\xi} \mathcal{P}_{\bar{\zeta}}=\frac{k}{2}
$$

with the general solution given by Eq. (9). The familiar form of this equation $Q_{\xi \bar{\xi}}+(k / 2) e^{2 Q}=0$ is obtained through the substitution $\mathscr{P}=e^{-Q}$. The only nonzero component of curvature is

$$
\Psi_{4}=-\frac{1}{u \mathcal{P}}\left(\mathscr{P} \mathcal{P}_{\zeta \zeta v}-\mathcal{P}_{\zeta \zeta} \mathcal{P}_{v}\right)
$$

and the solution is therefore Petrov type $N$. When we substitute for $h$ from Eq. (10) we find

$$
\Psi_{4}=-\frac{1}{u \mathcal{P}}\left(\mathcal{P P}_{\zeta \xi \xi}-\mathscr{P}_{\xi \xi} \mathcal{P}_{\xi}\right) \theta(v),
$$

which makes manifest the shock-wave character of this solution.

We conclude that the continuous metric (8) is Ricci flat, the hypersurfaces $v=$ const are shear-free cones, the Petrov type is $N$ and the Weyl tensor suffers a step discontinuity. Thus it satisfies all the requirements of an exact spherical shock-wave solution of the Einstein field equations.

\section{ACCELERATING SOLUTIONS}

The construction of the spherical shock wave solution in Sec. II is based on the identification of two pieces of Minkowski space with metric (5) across $v=0$ according to Eq. (6). In this process we have used the fact that the coordinate system employed in the metric (5) is such that the hypersurfaces $v=$ const are null cones. New shockwave solutions which include extra parameters should result if we can apply the same process of identification with warp to Minkowski space with another form of the metric, provided that null hypersurfaces $v=$ const are again cones. The metric for constant acceleration [17] is a case in point.

If we start with the Minkowski metric (1) and apply the transformation

$$
\begin{aligned}
v^{\prime} & =-\frac{1}{a}\left(e^{-a v}-1\right)+\frac{u}{P} e^{-a v}|\zeta|^{2}, \\
u^{\prime} & =\frac{k}{2 a}\left(e^{a v}-1\right)+\frac{u}{P} e^{a v}, \\
\zeta^{\prime} & =\frac{u}{P} \xi,
\end{aligned}
$$

where $a$ is a constant, the acceleration parameter, we find that the Minkowski metric becomes

$$
\begin{aligned}
d s^{2}= & 2 d u d v+\left(k+2 a \frac{u}{P}\right]\left[1-a \frac{u}{P}|\zeta|^{2}\right] d v^{2} \\
& +2 a \frac{u^{2}}{P^{2}}(\bar{\zeta} d \zeta+\zeta d \bar{\zeta}) d v-2 \frac{u^{2}}{P^{2}} d \zeta d \bar{\zeta}
\end{aligned}
$$

which reduces to Eq. (5) in the limit $a \rightarrow 0$. In this case $v$ is again null and both Eqs. (5) and (18) have the same degenerate metric on the hypersurfaces $v=$ const. So we can identify with warp, as in Eqs. (6), two pieces of Minkowski space with metric (18) across $v=0$. The result of this process is a new exact solution of the Einstein field equations with the null coframe

$$
\begin{aligned}
l= & d v, \\
n= & \mathcal{P} d u+\frac{1}{2}\left[k+2 a \frac{u}{\left|h_{\zeta}\right|}\right]\left(1-a \frac{u}{\left|h_{\zeta}\right|}|h|^{2}\right] d v \\
& +\left[\left(u \mathcal{P}_{\zeta}+2 a \frac{u^{2}}{\left|h_{\zeta}\right|^{2}} \bar{h} h_{\zeta}\right] d \zeta+\text { c.c. }\right], \\
m= & u d \zeta .
\end{aligned}
$$

where c.c. denotes the complex conjugate. Working out the curvature of this metric we find that it is a shear-free, type- $D$ solution of the vacuum Einstein field equations.

\section{ELECTROVAC SPHERICAL SHOCK WAVES}

The metric for the spherical shock wave solution (8) can be readily modified to yield an exact solution of the Einstein-Maxwell equations describing spherical gravitational and electromagnetic shock waves. We shall start with formally the same form of the metric as in Eq. (8) and suppose that the Maxwell potential one-form is given by

$$
A=(\mathcal{A}+\overline{\mathcal{A}}) d v
$$

where $\mathcal{A}$ is a holomorphic function

$$
\mathcal{A}_{\bar{\xi}}=0 \text {. }
$$

Then we find that the Einstein-Maxwell equations reduce to

$$
\mathcal{P P}_{\zeta \zeta \bar{\xi}}-\mathcal{P}_{\zeta \zeta} \mathcal{P}_{\overline{\xi \xi}}=-\kappa \mathcal{A}_{\zeta} \overline{\mathcal{A}}_{\bar{\xi}},
$$


where $\kappa$ is Newton's gravitational constant in geometrical units. We note that for $\mathcal{A}=0 \mathrm{Eq}$. (22) admits as first integral Liouville's equation (14), which is the vacuum field equation. The $v$ dependence of $\mathcal{A}$ in Eq. (23) must be chosen in the form

$$
\mathcal{A}=\mathcal{A}(\zeta+v \theta(v))
$$

to fit the similar dependence of $h$ in Eq. (10) so that Eq. (22), which contains no derivatives with respect to $v$, is consistent. In order to obtain an explicit solution we must specialize the functional form of $\mathcal{A}$ in Eq. (23) to any desired expression and find the corresponding expression for $h$ from Eq. (22).

\section{RELATIONSHIP TO ROBINSON-TRAUTMAN METRICS}

For spherical waves with smooth profiles the general form of the metric (8) is equivalent to the RobinsonTrautman solutions. We shall now demonstrate this equivalence by presenting the coordinate transformation which carries one form of the metric onto the other. $[6,7]$

Type- $N$ Robinson-Trautman solutions are given by

$d s^{2}=2 d U d V+C d V^{2}-d l^{2}$,

$d l^{2}=\frac{U^{2}}{P^{2}}\left\{\left[d X-Q_{Y} f(V) d V\right]^{2}+\left[d Y-Q_{X} f(V) d V\right]^{2}\right\}$,

$C=k+2 u\left[Q_{X Y}-\frac{1}{2 P}\left(X Q_{Y}+Y Q_{X}\right)\right] f(V)$,

$P=1+\frac{k}{4}\left(X^{2}+Y^{2}\right)$,

where $f$ is an arbitrary function describing the degree of freedom of the gravitational field and $Q$ is a harmonic function:

$$
Q_{X X}+Q_{Y Y}=0 \text {. }
$$

As we have remarked in the introduction, the metric (24) becomes meaningless if we were to allow $f$ to be a distribution. So $f$ must be $C^{0}$ in Eq. (24) and this rules out the possibility of representing impulsive, or shock waves by the Robinson-Trautman form because the Weyl tensor is proportional to $f$.

The key to finding the required transformation lies in the two-metric $d l^{2}$. If we rearrange the terms by completing the square for the coordinate differential $d V$, we find that $d l^{2}$ becomes isotropic if we can carry out a change of coordinates satisfying

$$
\begin{aligned}
& d x=\frac{Q_{X} d Y+Q_{Y} d X}{Q_{X}^{2}+Q_{Y}^{2}}-f(V) d V \\
& d y=\frac{Q_{X} d X-Q_{Y} d Y}{Q_{X}^{2}+Q_{Y}^{2}} \\
& d v=d V
\end{aligned}
$$

which does not involve $U$. A priori there is no guarantee that Eqs. (26), which are differential equations, will admit solutions for the functions $x, y$, and $v$. However, the integrability conditions obtained by applying the exterior derivative to Eqs. (26) are satisfied by virtue of Eq. (25). Since we are only interested in establishing a new local coordinate chart we can use Poincaré's lemma. Thus given any harmonic function $Q$ Eqs. (26) will admit a solution for the new coordinates. The remaining coordinate transformation

$$
u=\frac{U}{P}\left(Q_{X}^{2}+Q_{Y}^{2}\right)^{1 / 2}
$$

follows readily from the expression for conformal factor of the isotropic form of $d l^{2}$. Finally we let

$$
\xi=\frac{1}{\sqrt{2}}(x+i y)
$$

to obtain the metric (8) from Eqs. (24).

An alternative coordinate system for RobinsonTrautman solutions has been discussed by Foster and Newman [9]. For type- $N$ solutions this is given by

$$
d s^{2}=2 d u^{\prime} d v-2 u^{\prime}(\ln P)_{v} d v^{2}-2 \frac{u^{\prime 2}}{P^{2}} d \zeta d \bar{\zeta},
$$

where the definition of $P$ is the same as in Eq. (9). Equation (29) is also unacceptable as a shock wave metric because it is not continuous across $v=0$. The discontinuity is hidden in the derivative with respect to $v$ that appears in the coefficient of $d v^{2}$. If the dependence of $h$ on $v$ had been given by a differentiable function, rather than Eq. (10) which is the required form for a shock wave, then we could have readily transformed the Foster-Newman form of the metric to the form of Eq. (8) above by letting

$$
u^{\prime}=u P \text {. }
$$

It is worthwhile to remark again that the principal issue that is addressed in this paper is the construction of a coordinate system so that spherical shock waves will be described by a continuous metric. For waves with smooth profiles the various forms of the metric in Eqs. (24), (29), and (8) are completely equivalent, namely, type- $N$ Robinson-Trautman solutions. But for shock waves the particular coordinate system in which this solution is expressed is of paramount importance. Equations (24) and (29) are discontinuous across $v=0$ and therefore cannot be acceptable as metrics. Only Eq. (8) satisfies all the requirements of a pure spherical shock wave.

\section{CONCLUSION}

We have presented exact spherical shock wave solutions of the Einstein field equations. They emerge in a remarkably simple way through Penrose's approach of identification with warp.

\section{ACKNOWLEDGMENTS}

I thank Roger Penrose for many stimulating discussions. I thank also The British Council for a grant-in-aid. This work was supported in part by The Turkish Science Research Council TÜBİTAK. 
[1] R. Penrose, in General Relativity, Papers in Honour of J. L. Synge, edited by L. O'Raifertaigh (Clarendon, Oxford, 1972).

[2] K. A. Khan and R. Penrose, Nature (London) 229, 185 (1971).

[3] Y. Nutku and M. Halil, Phys. Rev. Lett. 39, 1379 (1977).

[4] P. Szekeres, J. Math. Phys. 13, 286 (1972).

[5] S. Chandrasekhar and B. Xanthopoulos, Proc. R. Soc. London A408, 175 (1986).

[6] I. Robinson and A. Trautman, Phys. Rev. Lett. 4, 431 (1960).

[7] I. Robinson and A. Trautman, Proc. R. Soc. London A265, 463 (1962).

[8] E. T. Newman and T. W. J. Unti, J. Math. Phys. 3, 891 (1962).
(1962)

[9] J. Foster and E. T. Newman, J. Math. Phys. 8, 189 (1967).

[10] R. Penrose and M. MacCallum, Phys. Rep. 6C, 241 (1972).

[11] Y. Nutku and R. Penrose (unpublished).

[12] T. W. B. Kibble, J. Phys. A 9, 1398 (1976).

[13] A. Vilenkin, Phys. Rep. 121, 263 (1985).

[14] M. Hortaçsu, Class. Quantum Grav. 7, L165 (1990).

[15] R. Gleiser and J. Pullin, Class. Quantum Grav. 6, L141 (1989).

[16] E. T. Newman and R. Penrose, J. Math. Phys. 3, 566 (1962).

[17] W. Kinnersley and M. Walker, Phys. Rev. D 2, 1359 (1970). 